\title{
Effect of an Argon Plasma Produced At The Atmospheric Pressure On Blood Coagulation And Wounds Healing
}

\author{
Hamid H. Murbat, Feza Shalal, Neda, Neean F. Mageed, Raghda H. Hassan, \\ Nisreen K. abdalameer, Sara Talal
}

\begin{abstract}
In this paper the study of the effects of cold Argon plasma in blood coagulation and wound healing was done. The group of different male of mice with two month age was chosen. Every mouse was cut by $1 \mathrm{~cm}$ piece of wound by knife in the rear of the back area. The studying includes two parts: the first, were done by chosen group healthy mice, and they classified into three subgroups the first was lefts without exposure to cold argon plasma (control), the second was exposure to 5 minute of plasma and the third was exposure to 15 minute of 5 discrete interval plasma exposure, the periods of every interval was 3 minute. It is found that the cold argon plasma have big positive effects on the blood coagulation processing and wound healing, and its effecting improves with increasing of time of exposures. The second parts of the study concerned cold plasma in the treatment of wounds in mice suffers increasing in the blood sugar concentration, where the mice were injected by $0.1 \mathrm{ml}$ of glucose. The wound of mice was exposure to 6 minute of cold plasma. Also, It is found that the cold argon plasma have big positive effects in processing and wound healing in mice suffers increasing in the blood sugar concentration.
\end{abstract}

Keywards: cold Argon plasma, blood coagulation, wound healing.

\section{Introduction}

Plasma produced by electric discharge is classified into two types, thermal and non-thermal plasma. Thermal plasma has electrons with a temperature is approximately equal to the other plasma components (Ions and neutral particles), i.e. $\mathrm{T}_{\mathrm{e}} \approx \mathrm{T}_{\mathrm{i}} \approx \mathrm{T}_{\mathrm{n}}$. On the other hand, non-thermal plasma, which is also called cold plasma, has electrons with a temperature significantly higher than that of other plasma components, which is equal to room temperature i.e. $\left(\mathrm{T}_{\mathrm{e}} \gg \mathrm{T}_{\mathrm{i}} \approx \mathrm{T}_{\mathrm{n}} \approx 300\right)[1]$.

Both types of plasmas have many and different important applications. Because of its high temperature, thermal plasma is used (a) in the process of cutting and welding minerals [2], (b) in the treatment of solid municipal waste, and medical, industrial, and nuclear waste to convert them to their basic contamination [3]. In contrast, and of the environmental and living pollutants [4-7], (b) in medical applications such as sterilizing instruments and medical devices [8], dental care [9], treatment of skin diseases and some cancers[ 10], wound healing [11], and anticoagulation [12].Medical treatment of cold plasma is performed through the interaction of plasma with living tissue by two methods [13]:

First, direct method in this method, the body of the organism represents one electrode and most of the current passes through its body. The direct method allows for plenty of active particles, which were generated by plasma, such as atoms, molecules, and ultraviolet radiation to reach the living tissue and interact with it. These active particles include ozone $\mathrm{O}_{3}, \mathrm{NO}$, and $\mathrm{OH}$ free radicals, but the main feature of the direct method its containment of plenty of charged particles such as electrons, negative, and positive ions that can reach the living tissue [13]. Second, indirect method in this method, the body of the organism is between the two electric electrodes that generate the plasma. This method includes the influx of non-charged atoms and molecules, which were generated in the plasma particles, with a small number of charged particles that reach the surface of living tissues.In both methods, the characteristics of plasma can be controlled via the control of the concentrations of the plasma components[13]. The fact that the direct method of plenty of charged particles gives the possibility to control the effects of non-thermal plasma. On the other hand, the importance of the indirect method lies when the treated surface is relativelyat a far distance from the plasma device [13]. The nitric oxide NO that is generated in the plasma proved that is highly effective in closing the wounds and accelerating the mechanism of wounds healing [14.15-17]. In the present study, in vivo effect on wounds healing was investigated. A group of mice was wounded and then exposed to plasma to observe temporal evolution of their recovery.

\section{Materials and Methods}

\section{1- Plasma system}

The homemade plasma jet was used for the production of non-thermal argon plasma. The system consist of two concentrated cylinders, the first cylinder is made of glass $100 \mathrm{~mm}$ long has an internal diameter of $10 \mathrm{~mm}$. The second cylinder is made of copper $90 \mathrm{~mm}$ long has an internal diameter of $3 \mathrm{~mm}$. The copper 
cylinder extends along the glass cylinder except for a distance of $10 \mathrm{~mm}$ at the end to be a plasma production area. The internal copper cylinder works as a guide for gas, and this cylinder is connected to the anode which is one of the electrodes of high power source whereas the second electrode (ground electrode) of high power source is connected to the ring surrounded the glass cylinder from the outside near its end. Argon gas is used to feed the system, the average), the peak of the voltage of the high power source is $25 \mathrm{kV}$, and the frequency is 30 $\mathrm{kHz}$. In the present study, the voltage was chosen to be $5 \mathrm{kV}$ and the frequency is $22 \mathrm{kHz}$. Figure 1 shows the photographic image of the plasma system that is used in the treatment of wounds in mice.

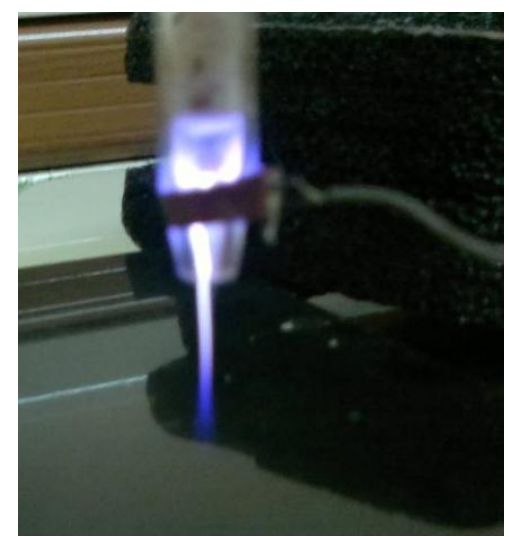

Figure (1): Plasma system at operation.

\section{2- Mice preparing}

In the current study, a group of mice was selected from both sexes and ages two months to study the effect of cold plasma on wounds healing. The study was divided into two parts: the first part of the study included the effect of cold plasma on the wounds healing of normal mice which do not suffer from the high rate of diabetes in the blood, and the second part of the study included the effect of the plasma on the wounds healing of abnormal mice which suffer from the high rate of diabetes in the blood.

\section{3- Study the effect of cold plasma on wounds healing in normal mice}

The group of mice was divided into three subgroups which are

a- Control group:

a group of normal mice represents control group, each mouse was shaved in the area where the wound will be. A scalpel was used to make a wound of $1 \mathrm{~cm}$ long in the rear area of the dorsal of each mouse as can be seen in Figure (2). One of the wounded mice was left without exposure to the cold plasma to serve as control to observe the time of the wound case and the recovery time. It was found that the control mouse has died after 24 hours due the bleeding. It was observed that most of the control mice die after different periods of time because the bleeding from the wound.

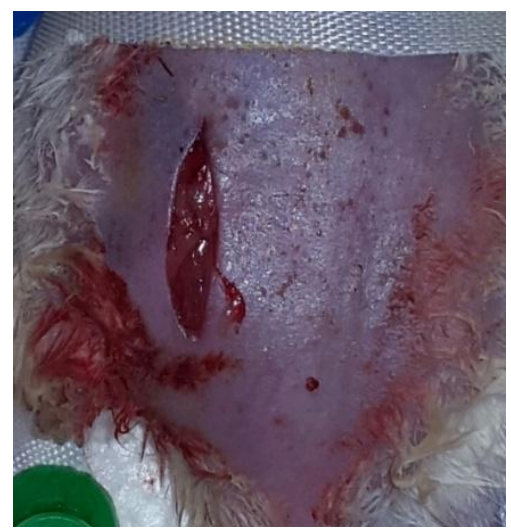

Figure (2): Wounded mice before exposure to the cold plasma.

\section{B) Second group}

This group was exposed to cold plasma for 5 minutes, the time evolution of the wound case and recovery time was recorded. Figure $(3, a, b, c)$ shows the wound case after 5, 12, 30 minutes respectively after the exposure. It was found that the wounded mice which were exposed to plasma for 45 minutes recovered and the wound case improved after short time follows the exposure as can be seen in figure 3 . 


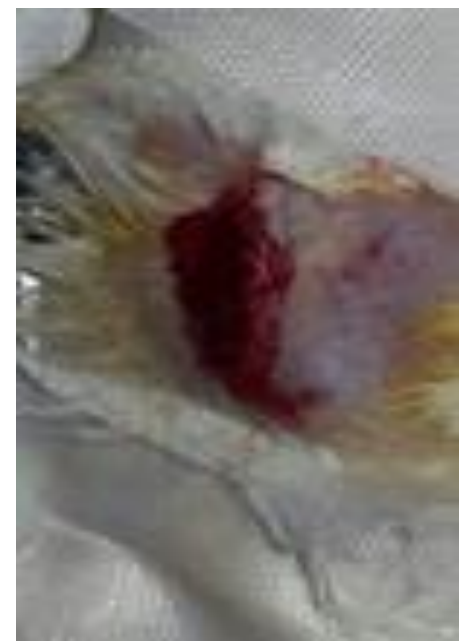

Figure (3-a): Wounded mice directly after exposure for 5 minutes.

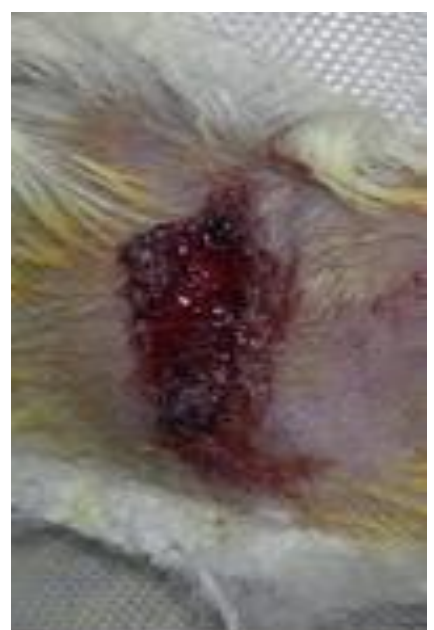

Figure (3-b): Wounded mice after 12 minutes when exposure for 5 minutes.

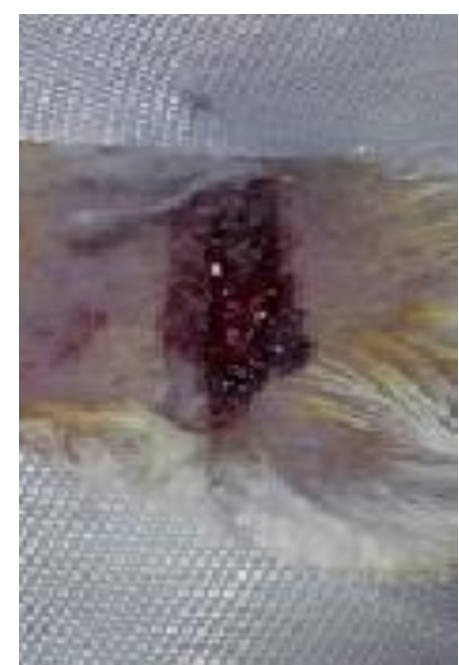

Figure (3-c): Wounded mice after 15 minutes when exposure for 5 minutes.

\section{c) Third group}

This group was exposed to the cold plasma for 15 minutes at 5 discrete periods; the time of each period was 3 minutes. The time evolution of the wound case and recovery time was recorded. Figure (4-a,b,c,d,e,f) shows the wound case after each period of exposure. 


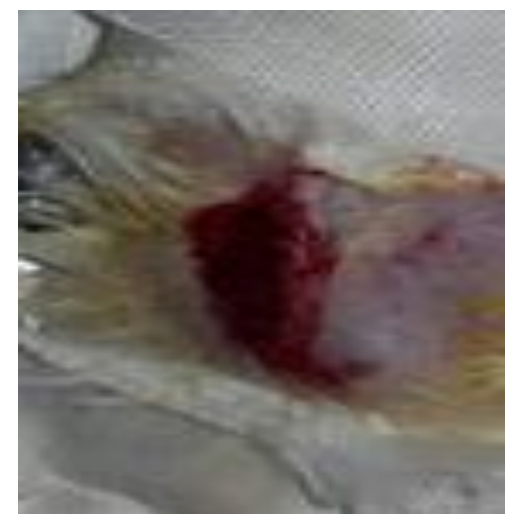

Figure (4-a): Wound case before exposure.

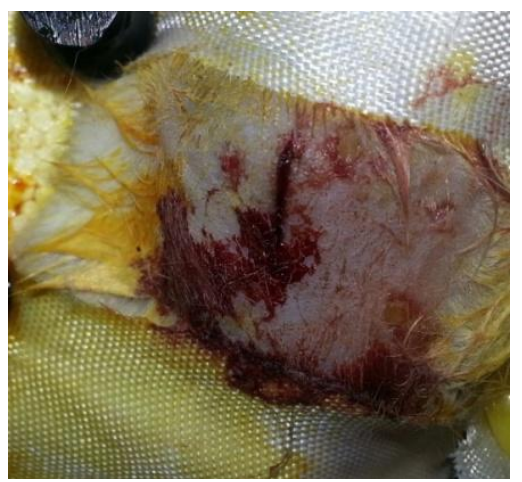

Figure (4-b): Wound case directly after exposure for 3 minutes.

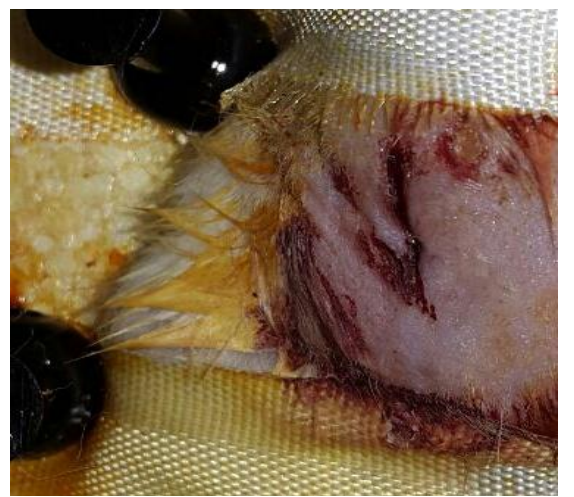

Figure (4-c): Wound case directly after exposure for 6 minutes.

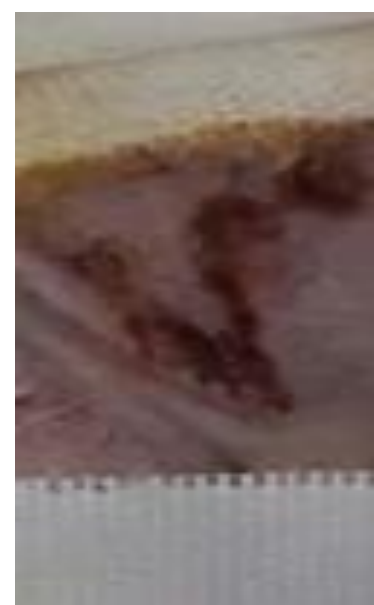

Figure (4-d): Wound case directly after exposure for 9 minutes. 


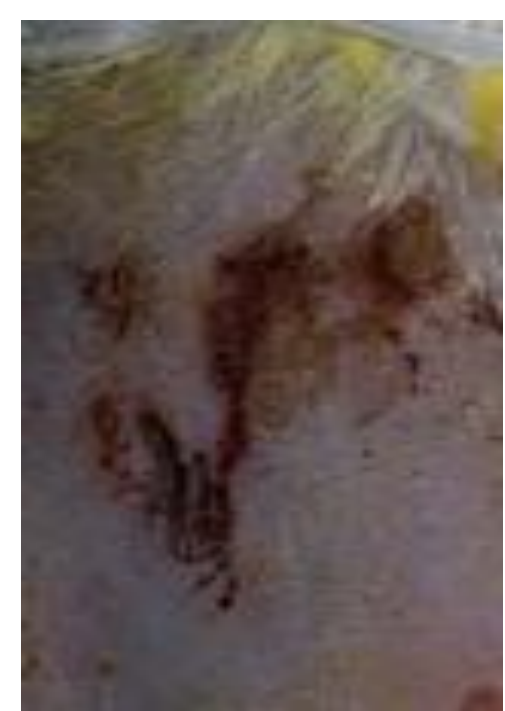

Figure (4-e): Wound case directly after exposure for 12 minutes.

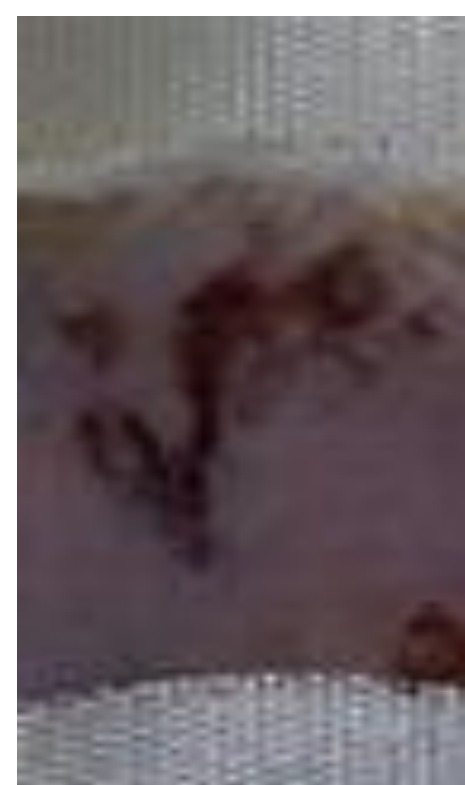

Figure (4-f): Wound case directly after exposure for 15 minutes.

from the current results of this experiment in which the time of exposure was increased and was given in different sessions separated by regular intervals indicated that it is possible to increase the time of exposure by dividing the total time of exposure to short and separated sessions, and this would help to accelerate the healing of wounds, and did not cause any noticed side effects as a result of increased exposure.

\section{4- Study the effect of cold plasma on wounds healing in abnormal mice which suffer high blood sugar (diabetic)}

In order to study the effect of the cold plasma on wound healing in mice which suffer high blood sugar, the wounded mice were injected with $0.1 \mathrm{ml}$ Glucose solution. After that, the wound of each mouse was exposed to cold plasma for 6 minutes as two sessions in which the time of each session was 3 minutes. The interval between the two sessions was 15 minutes. Figure( 5- a, b, c, d, e) shows photos of the wound of the mouse, which suffers an increase in blood sugar.It is known that the increase in blood sugar greatly reduces the process of blood coagulation and delays wound healing processes. Through the practical results conducted on the wounded mice suffering an increase blood sugar, it was found that cold plasma has a significant positive impact of helping in the blood coagulation process and the healing of wounds as can be shown in figure (-5 a, b, $\mathrm{c}, \mathrm{d}, \mathrm{e})$. 


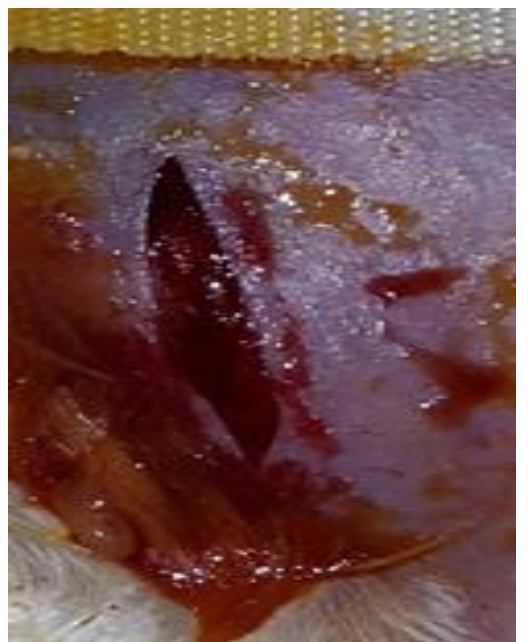

Figure (5-a): Wound after exposure (mice were injected Glucose solution).

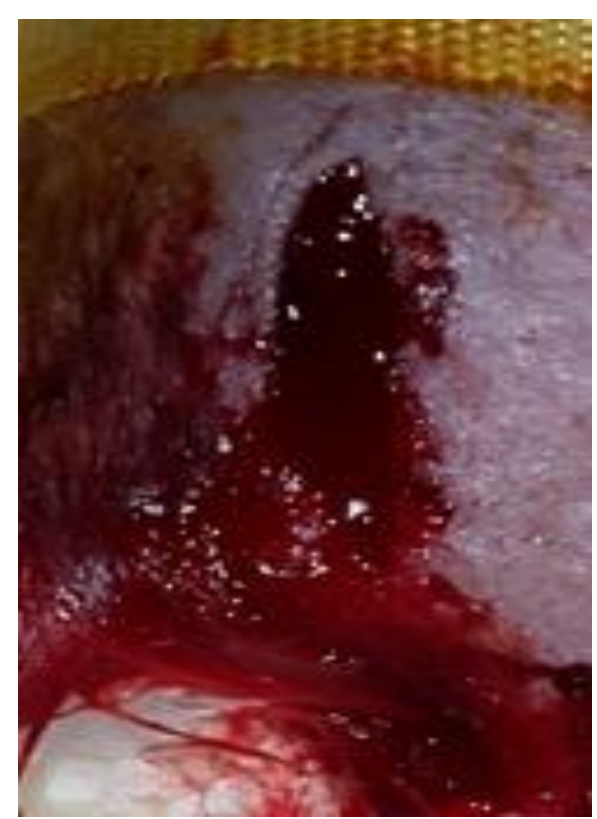

Figure (5-b): Wound directly after exposure for 3 minutes.

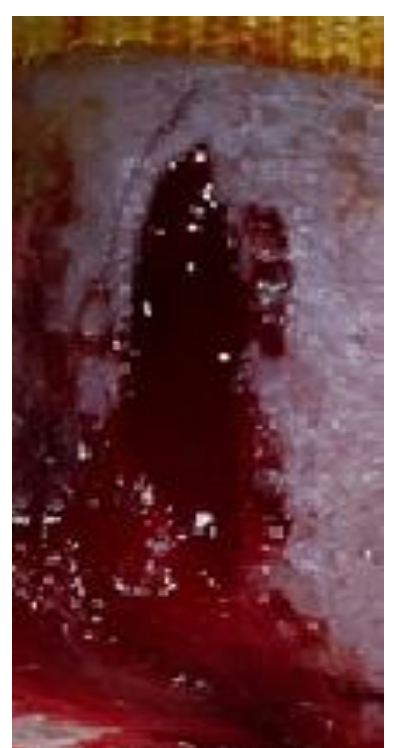

Figure (5-c): Wound directly after exposure for 6 minutes. 


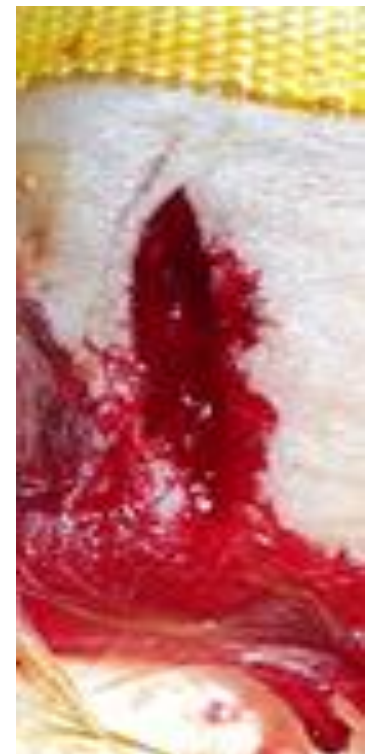

Figure (5-d): Wound after 15 minutes after exposure for 6 minutes.

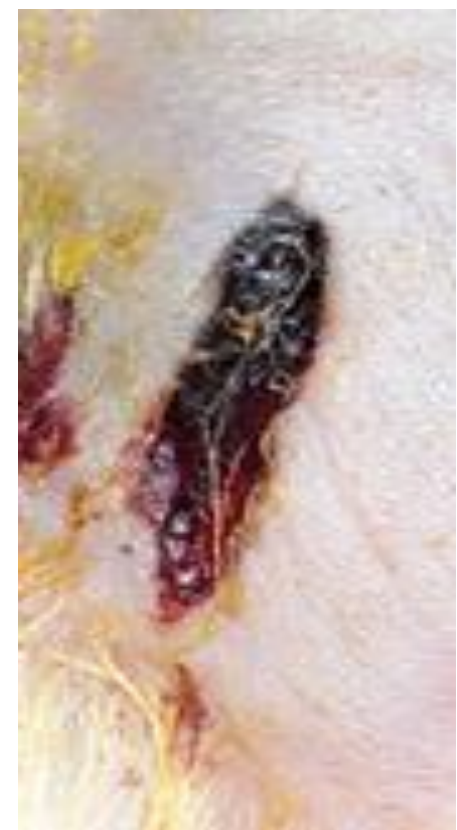

Figure (5-e): Wound after 1day after exposure for 6 minutes.

\section{Conclusions}

The current study was performed to investigate the effect of cold plasma on the blood coagulation process and wounds healing. The experiments were achieved on a group of mice. The study included two parts: the first part was done to study the effect of cold plasma on wounds in normal mice, and the second is to study the effect of the plasma on wounds in mice suffer high blood sugar in their blood.

For normal mice, it has proven that cold plasma has a significant positive impact on the blood coagulation process and the healing of wounds. And it was found the effect of plasma to heal the wounds improves as the exposure time increases. The results of the second part, which included the effect of the plasma on the wounds healing in mice suffer high blood sugar, showed that the cold plasma has a significant positive impact on helping in the process of blood coagulation and wound healing.

\section{References}

[1]. T. J. M. Boyd, and J. J. Sanderson, "The physics of plasmas", Cambridge University Press, Cambridge (2003)

[2]. I. Bica, "The Physics and the Technology of Materials in Plasma", Mirton Press, Timisoara (2005)

[3]. Vijay Neha, International Journal of Engineering, Volume (2) : Issue (1)

[4]. R. Hippler, S. Pfan, and M. Schmidt, "Low temperature plasma physics: fundamentalaspects and applications", Wiley VCH, Berlin (2001) 
[5]. J. Reece Roth, "Industrial Plasma Engineering, vol. 2; Applications to nonthermal plasmaprocessing", IOP Publishing Ltd, Cornwall, UK (2001)

[6]. R. d'Agostino, P. Favia, Y. Kawei, H. Ikegami, N. Sato, and F. Arefi-Khonsari, “Advanced Plasma Technology”, Wiley-VCH VerlagGmbH\&Co K Cron A, Weinheim (2008)

[7]. M. Thiyagarajan, I. Alexeff, S. Parameswaran, and S. Beebe, IEEE Transactions on Plasma Science, 33 (2005) $322-323$

[8]. Grundmann H, Aires-de-Sousa M, Boyce J and Tiemersma E 2006 Emergence and resurgence of meticillinresistantStaphylococcus aureusas a public-health threat Lancet 368 874-85

[9]. Klein L and Gibbs R 2004 Use if microbial cultures and antibiotics in the prevention of infection-associated preterm birth AJOG $1901493-502$

[10]. AkramMohammadi Nokhandani1, SyedeMahsaTaheri Otaghsara1, MahboubehKhadem Abolfazli1 , Masoumeh karimi1, Fereshteh Adel 1, Hamed babapour2, Gholamreza Atae1* , Cancer Treatment, Sch. Acad. J. Biosci., 2015; 3(2B):222-230

[11]. Etufugh C N and Phillips T J 2007 Venous ulcers Clin. Dermatol.25 121-30

[12]. Gregory Fridman · Marie Peddinghaus · HalimAyan · Alexander Fridman, Blood Coagulation and Living Tissue Sterilization by Floating-Electrode Dielectric Barrier Discharge in Air, Plasma Chem Plasma Process , DOI 10.1007/s11090-006-9024-4

[13]. Fridman, G., et al., Comparison of Direct and Indirect Effects of Non-Thermal Atmospheric Pressure Plasma on Bacteria. Plasma Processes and Polymers, 2006: p. 10.1002/ppap.200600217, accepted.

[14]. Fridman G, Shekhter AB, Vasilets VN, Friedman G, Gutsol A, Fridman A. Applied plasma medicine. Plasma Process Polym. 2008;5:503-533.

[15]. Gostev V. Cold plasma in biological investigations. In: Proceedings of the NATO Advanced Study Institute (ASI): Plasma Assisted Decontamination of Biological and Chemical Agents, Çeşme, 2007. Misyn FA, Gostev VA. "Cold” plasma application in eyelid phlegmon curing. In: Diagnosis and treatment of infectious diseases. Petrozavodsk, Russia; 2000.

[16]. Kong M, Kroesen G, Morfill G, Nosenko T, Shimizu T, Dijk J, Zimmermann J. Plasma medicine: an introductory review. New J Phys. 2009; 11:115012. 